\title{
Homomorphic wavelet shrinkage and feature emphasis for speckle reduction and enhancement of echocardiographic images
}

\author{
Xuli Zong \\ Department of Computer \& Information Science \& Engineering \\ University of Florida, Gainesville, FL 32611 \\ Edward A. Geiser \\ Echocardiography Research Laboratory, Department of Medicine \\ University of Florida, Gainesville, FL 32610 \\ Andrew F. Laine \\ Department of Computer \& Information Science \& Engineering \\ University of Florida, Gainesville, FL 32611 \\ David C. Wilson \\ Department of Mathematics \\ University of Florida, Gainesville, FL 32611
}

\begin{abstract}
An approach for speckle reduction and feature enhancement under a framework of multiscale wavelet analysis is presented. The advantages of both soft thresholding and hard thresholding wavelet shrinkage techniques are utilized to eliminate noise and preserve the sharpness of salient features. We integrate a method of wavelet shrinkage with nonlinear processing to enhance contrast within structures and along object boundaries. Feature restoration and enhancement are accomplished by modifying the gain of a signal's variational energy. In this study, we focus on multiplicative noise, such as speckle noise. We show that this algorithm is capable of enhancing features of interest, such as endocardial and epicardial boundaries in 2-D short-axis echocardiograms while at the same time reducing speckle.

Speckle is modeled as multiplicative noise, and approximated by additive stationary Gaussian white noise on a logarithmic scale. In our algorithm, shrinkage of wavelet coefficients via soft thresholding within finer scales is carried out on coefficients of logarithmically transformed echocardiograms. Nonlinear feature emphasis combined with hard thresholding within middle levels of transform space is performed subsequently on wavelet coefficients. The first operation reduces speckle while the second step accomplishes structure and object boundary enhancement.

Preliminary results suggest that the algorithm can remove speckle noise and enhance contrast along boundaries of importance to cardiologist. We have applied this algorithm to echocardiograms of varying quality, and present both experimental analysis and sample results.
\end{abstract}

Keywords: wavelet transform, wavelet shrinkage, feature emphasis, speckle reduction, contrast enhancement

\section{INTRODUCTION}

Signal and image degradation by noise and artifacts is a phenomena of acquisition in the real world. Different image modalities exhibit distinct types of degradation. Radiographs often have low contrast while images formed with coherent energy, such as ultrasound, suffer from speckle noise. Image degradation can have a significant impact on human interpretation and the development of computer-assisted methods. Noise and artifacts often make feature 
extraction, analysis, recognition, and quantitative measurements algorithms unreliable. In this paper, de-noising and feature enhancement techniques are proposed to assist in the reliability of image processing algorithms for quantification.

Formation of images using coherent waves results in a granular pattern known as speckle. The granular pattern is correlated with the surface roughness of an object being imaged. Goodman ${ }^{10}$ presented an analysis of speckle properties under coherent irradiance, including laser. The primary differences between laser and ultrasound speckle were pointed out by Abbott and Thurstone ${ }^{1}$ in terms of coherent interference and speckle production. For speckle reduction, earlier techniques include temporal averaging, ${ }^{10,1}$ median filtering, and homomorphic Wiener filtering. ${ }^{13}$ Similar to temporal averaging, one speckle reduction technique ${ }^{23}$ uses frequency and/or angle diversity to generate multiple uncorrelated synthetic-aperture radar images which are summed incoherently to reduce the speckle. Homomorphic Wiener filtering is a method which converts multiplicative noise into additive noise and applies Wiener low-pass filtering to reduce noise. In [12], a coherent image was decomposed into three components, one of which, called subresolvable quasi-periodic scatter, causes speckle noise. The component was eliminated by a harmonic analysis algorithm. In [11], a wavelet-based method for speckle reduction was reported, but is distinct from the homomorphic approach described here. An algorithm ${ }^{22}$ based on the maximum-likelihood principle and a wavelet regularization procedure for the logarithm of a radar image was also developed to reduce speckle. In the last two decades, many image enhancement methods have been developed and reported in the literature. ${ }^{13-16,19}$ Specifically, various spatial and frequency-based techniques ${ }^{4,13,8,17}$ have been developed for ultrasound image enhancement. A method called statistical enhancement ${ }^{9}$ uses the local standard deviation of a surrounding region centered around each pixel to replace its value to enhance edges in ultrasound images.

De-Noising and feature enhancement appear to be two conflicting objectives. The purpose of de-noising is to eliminate noise in high frequency while methods of feature enhancement attempt to enhance high frequency signal details. The difference is that features often have a wider frequency band than noise. It is even more difficult to achieve both objectives when signal details are corrupted by noise. Traditional spatial- and filtering-based methods for de-noising often reduce noise at the price of blurred features while single-scale conventional methods for feature enhancement may amplify noise. In our approach, we achieve de-noising and feature enhancement under a framework of multiscale wavelet analysis. We seek to eliminate noise while restoring or enhancing salient features. A discrete dyadic wavelet transform (DWT) with a first order derivative of a smoothing function as its basis wavelet can separate feature variational energy (VE) and noise VE in a wavelet domain reasonably well. The objectives of de-noising and feature enhancement can be achieved by simultaneously lowering noise VE and raising feature VE through judicious nonlinear processing of wavelet coefficients (WC) in the transform domain.

The organization of this paper is as follows. Section 2 presents the methodology for our de-noising and feature enhancement algorithm. It includes speckle noise modeling, finite-level discrete dyadic wavelet transform and its implementation based on two-level recursive relations. It also includes wavelet shrinkage for noise reduction, nonlinear feature emphasis for contrast restoration and enhancement, and the complete algorithm. In Section 3, we present experimental results and analysis. A study of clinical images has been conducted to test the effects of de-noising on the consistency and reliability to manually defined borders by expert observers. Quantitative measurements show that borders defined by experts on de-noised echocardiograms are more consistent than those on the corresponding speckled echocardiograms. Statistical results are also presented. For how these echocardiographic sequences were acquired, see reference. ${ }^{24}$ Section 4 concludes the paper and suggests some future directions of research.

\section{METHODOLOGY}

Its non-invasive nature, low cost, portability, and real-time image formation make ultrasound imaging an attractive means for medical diagnosis, especially in cardiology. One of the limitations of ultrasound images is speckle noise. Speckle reduction remains a difficult problem due to the lack of reliable models to estimate noise. Speckle under different imaging media, such as laser, radar, or ultrasound, may look slightly different. However the granular pattern in each of these is produced by coherent interference related to the roughness of object surfaces. An approximate speckle noise model ${ }^{13}$ is formulated here without temporal averaging. We apply the approximate speckle noise model 
under a framework of multiscale wavelet analysis for speckle reduction and feature enhancement in echocardiographic images. By incorporating a feature enhancement mechanism into a de-noising process, we are able to not only reduce noise, but also restore features. Since we are interested in image noise reduction and feature enhancement, the problems of de-noising and enhancement are directly formulated in two-dimensions.

\subsection{Approximate speckle noise model}

Jain ${ }^{13}$ presented a model for speckle noise as

$$
f(x, y)=g(x, y) \eta_{m}(x, y)
$$

where $g(x, y)$ is an unknown 2-D function, such as a noise-free original image, to be recovered, $f(x, y)$ is a noisy observation of $g(x, y), \eta_{m}(x, y)$ is multiplicative noise, $x$ and $y$ are the variables, such as spatial locations, and $(x, y) \in R^{2}$. To separate the noise from the original image, we take a logarithmic transform on the both sides of (1) and approximate $\eta_{m}(x, y)$ in a log scale as additive Gaussian white noise. We now have option to apply various multiresolution representations for additive noise reduction. Jain showed a similar homomorphic approach ${ }^{13}$ for speckle reduction of images of stationary and undeformable objects through temporal averaging or homomorphic Wiener filtering. The dynamic deformable nature of echocardiograms through time prevents us from getting the same status of the left ventricle for multiple frames. Since we treat noise and features differently, we are able to accomplish a superior result than de-noising alone algorithms.

\subsection{Discrete dyadic wavelet transform}

A dyadic wavelet transform was first presented by Mallat and Zhong $^{20}$ and has been a useful tool in various application areas, such as data compression, edge detection, texture analysis, noise reduction, and image enhancement. In general, a finite-level discrete dyadic wavelet transform of a 2-D discrete function $f(m, n) \in L^{2}\left(Z^{2}\right)$ can be represented as

$$
\mathcal{W}[f(m, n)]=\left\{\left(W_{j}^{d}[f(m, n)]\right)_{d=1,2,1 \leq j \leq J}, S_{J}[f(m, n)]\right\}
$$

where $W_{j}^{d}[f(m, n)]$ is some wavelet coefficient at scale $2^{j}$ (or level $j$ ), position $(m, n)$, and spatial orientation $d(1$ for horizontal and 2 for vertical), $S_{J}[f(m, n)]$ is a coarse scale approximation at the final level $J$ at position $(m, n)$. $d=1,2$ represent horizontal or vertical spatial orientation. $\left(W_{j}^{d}[f(m, n)]\right)^{2}$ is typically referred to as part of a signal's energy. $W_{j}^{d}[f(m, n)]$ reflects a signal's variation when a basis wavelet is the first-order derivative of a smoothing function. ${ }^{20}$ The local maxima of $W_{j}^{d}[f(m, n)]$ in terms of magnitude corresponds to sharp variation points in an image while the minima of $\left|W_{j}^{d}[f(m, n)]\right|$ indicate slow intensity variation. Thus we describe $\left(W_{j}^{d}[f(m, n)]\right)^{2}$ as a signal's variational energy (VE). The finite-level dyadic wavelet decomposition in (2) forms a complete representation for a J-level dyadic wavelet representation. For a particular class of 2-D dyadic wavelets, such as the first order derivatives of spline smoothness functions, Mallat and Zhong ${ }^{20}$ showed that the finite-level direct and inverse discrete dyadic wavelet transform of a 2-D discrete function can be implemented in terms of four FIR filters, $H, G, K$, and $L$. The dyadic wavelet decomposition in (2) can be formulated in terms of the following recursive relations between the two levels $j$ and $j+1$ in the Fourier domain as

$$
\begin{gathered}
\hat{W}_{j+1}^{1}\left[f\left(\omega_{x}, \omega_{y}\right)\right]=G\left(2^{j} \omega_{x}\right) \hat{S}_{j}\left[f\left(\omega_{x}, \omega_{y}\right)\right], \\
\hat{W}_{j+1}^{2}\left[f\left(\omega_{x}, \omega_{y}\right)\right]=G\left(2^{j} \omega_{y}\right) \hat{S}_{j}\left[f\left(\omega_{x}, \omega_{y}\right)\right], \text { and } \\
\hat{S}_{j+1}\left[f\left(\omega_{x}, \omega_{y}\right)\right]=H\left(2^{j} \omega_{x}\right) H\left(2^{j} \omega_{y}\right) \hat{S}_{j}\left[f\left(\omega_{x}, \omega_{y}\right)\right],
\end{gathered}
$$

where $j \geq 0$, and $\hat{S}_{0}\left[f\left(\omega_{x}, \omega_{y}\right)\right]=\hat{f}\left(\omega_{x}, \omega_{y}\right)$. The reconstruction $\hat{S}_{0}\left[f\left(\omega_{x}, \omega_{y}\right)\right]$ from a dyadic wavelet decomposition can be implemented through the inverse process of the above recursive decomposition. The DWT decomposition and reconstruction based on the above recursive relations are shown schematically in Figure 1.

\subsection{Wavelet shrinkage and feature emphasis}

After converting multiplicative noise into additive noise through a log homomorphic transform, we apply various new-emerging de-noising techniques ${ }^{7,6,2,3,18,21}$ to eliminate noise. We also apply methods of contrast enhance- 


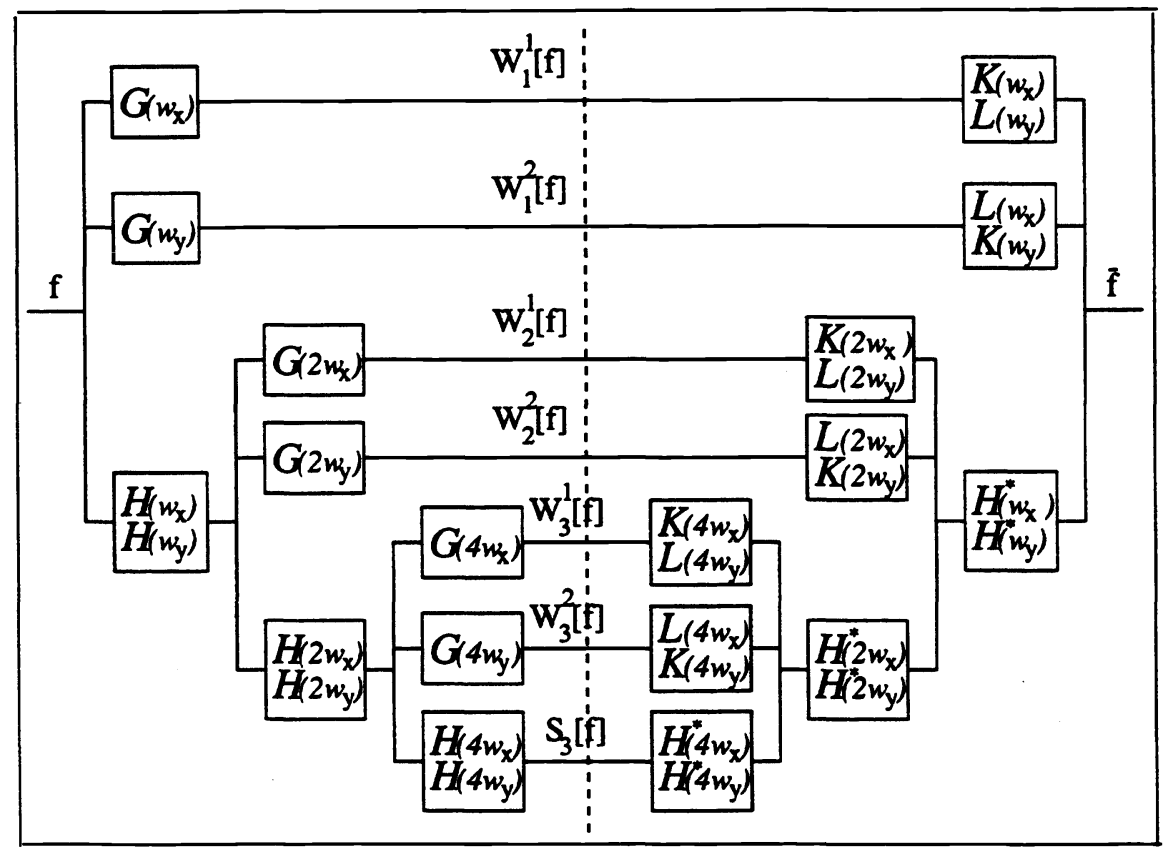

Figure 1: A 3-level DWT decomposition and reconstruction of a 2-D function.

ment ${ }^{14,19,15,16}$ to enhance cardiographic features. To achieve both objectives, we need (1) a representation which can separate features from noise and (2) effective de-noising and feature enhancement techniques. In this paper, we have investigated hard thresholding and Donoho's soft thresholding wavelet shrinkage for speckle reduction on a logarithmic scale. An advantage of soft thresholding is that it provides smoothness while hard thresholding preserves features. In our approach, we take advantage of both thresholding methods. Donoho's soft thresholding method ${ }^{6}$ was developed on an orthonormal wavelet transform, ${ }^{5}$ with Daubechies's Symmlet 8 basis wavelet. Previous de-noising results have shown some undesired side-effects, including pseudo-Gibbs phenomena. ${ }^{3}$ By using DWT and an antisymmetric basis wavelet without any oscillations, we are relatively free from such side-effects after de-noising. Our experiments show that a DWT with a first-order derivative of a smoothing function as its basis wavelet can separate feature VE and noise VE in the transform domain reasonably well.

\subsubsection{Wavelet shrinkage by soft thresholding}

A soft thresholding ${ }^{6}$ operation can be represented as

$$
u(\mathbf{x})=\mathcal{T}(v(\mathbf{x}), t)=\operatorname{sign}(v(\mathbf{x}))(|v(\mathbf{x})|-t)_{+}
$$

where the threshold parameter $t \propto \sigma$ noise level, $\mathbf{x} \in D$, the domain of $v(\mathbf{x})$, and $u(\mathbf{x})$ is the result of soft thresholding and has the same sign as $v(\mathbf{x})$ if non zero. DWT coefficients can be modified for noise reduction through this operation. Donoho's method of soft thresholding uses a single global threshold. Since noise coefficients within a DWT have average decay through fine-to-coarse scales, we regulate soft thresholding wavelet shrinkage by applying coefficient dependent thresholds at distinct scales.

\subsubsection{Feature emphasis by generalized adaptive gain}

Adaptive gain nonlinear processing ${ }^{14-16}$ has been successfully used to enhance features in digital mammography. Here, adaptive gain nonlinear processing is generalized to incorporate hard thresholding to avoid amplifying noise and remove small noise perturbations within middle scales. A generalized adaptive gain (GAG) nonlinear operator 
is defined as

$$
E_{G A G}(v)= \begin{cases}0 & |v|<T_{1} \\ \operatorname{sign}(v) T_{2}+\bar{a}(\operatorname{sigm}(c(u-b))-\operatorname{sigm}(-c(u+b))) & T_{2} \leq|v| \leq T_{3} \\ v & \text { otherwise }\end{cases}
$$

where $v \in\left[\begin{array}{ll}-1 & 1\end{array}\right], \bar{a}=a\left(T_{3}-T_{2}\right), u=\operatorname{sign}(v)\left(|v|-T_{2}\right) /\left(T_{3}-T_{2}\right), b \in(01), 0 \leq T_{1} \leq T_{2}<T_{3} \leq 1, c$ is a gain factor, and $a$ can be computed as

$$
\begin{gathered}
a=\frac{1}{\operatorname{sigm}(c(1-b))-\operatorname{sigm}(-c(1+b))} \\
\operatorname{sigm}(v)=\frac{1}{1+e^{-v}} .
\end{gathered}
$$

$E_{G A G}(v)$ is simply an enhancement operator, and $T_{1}, T_{2}$, and $T_{3}$ are selected parameters. When $T_{1}=T_{2}=0$ and $T_{3}=1$, the formula is equivalent to a previous adaptive gain nonlinear operator. ${ }^{14-16}$ The interval $\left[T_{2}, T_{3}\right]$ serves as a sliding window for feature selectivity. It can be adjusted to emphasize features within a specific range of variation. By selecting a gain, a window, and other parameters, we achieve distinct enhancement effects. Thus, through this nonlinear operator, DWT coefficients are processed for feature enhancement.

\subsection{Overview of algorithm for speckle reduction and feature enhancement}

The complete algorithm is described by the following six major steps.

1. Perform a logarithmic transform to convert an image containing multiplicative noise into an image with additive noise.

2. Carry out a DWT and obtain a complete representation of the log-transformed image in the transform domain.

3. Shrink coefficients within the finer scales to partially remove noise variation.

4. Emphasize features through nonlinear point-wise processing to increase the energy among features within a specific range of variation.

5. Perform an inverse DWT and reconstruct the de-noised and enhanced image so that it approximates it's noise-free original in log scale.

6. Finally, perform an exponential transform on the reconstructed image to convert it from log scale to linear scale. The resulting image is now de-noised and enhanced.

Speckle noise is the result of coherent imaging. It occurs whenever the surface roughness of objects is in the order of the wavelength of coherent radiation, and is treated here as multiplicative noise. ${ }^{13}$ The log transforms used in Steps 1 and 6 separate noise from some desired object function. We then approximate the noise as additive stationary Gaussian white noise. In this study, we were interested in enhancing structural features, for example endocardial and epicardial boundaries in 2-D short-axis echocardiograms.

Steps 2 and 5 were used to carry out the decomposition and reconstruction of a DWT. The selected wavelet basis was smooth without oscillations. ${ }^{20}$ Thus smoothing can be achieved without sacrificing mean square error performance since the feature enhancement mechanism is in the de-noising process itself. The analysis and synthesis filters employed to perform the DWT have compact support of a few taps. ${ }^{20}$ The decomposition and reconstruction is carried out using efficient spatial 1-D horizontal and vertical based convolution operations, making real-time implementation feasible.

Steps 3 and 4 accomplish de-noising and contrast enhancement via wavelet shrinkage and feature emphasis. In our implementation, wavelet coefficients are first processed to eliminate noise VE and to add signal VE to features at scales which more reliably compensate the loss of VE during wavelet shrinkage. This is accomplished through the energy gain nonlinear operator defined by Equation (7). The justification for adopting regulated soft thresholding 


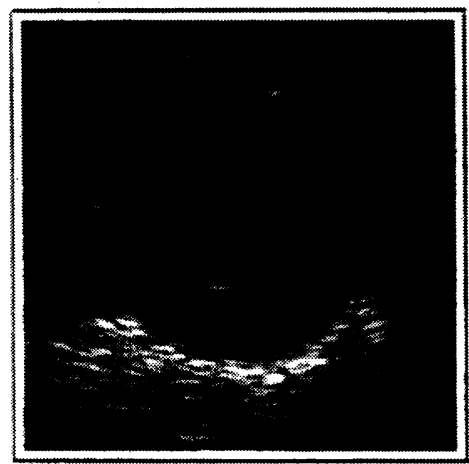

(a)

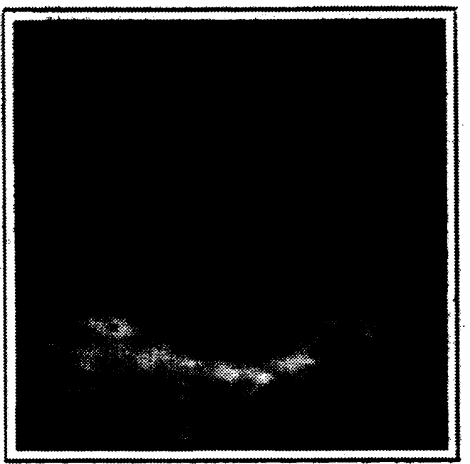

(b)

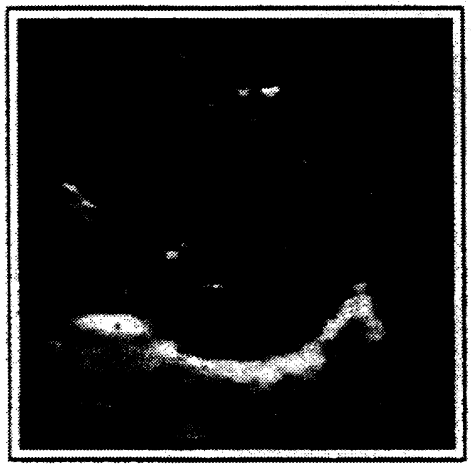

(c)

Figure 2: Results of de-noising and enhancement: (a) An noisy ED frame, (b) Wavelet shrinkage de-noising only method, and (c) DWT-based de-noising and enhancement.

is that noise VE in the transform domain decreases through fine-to-coarse scales. Regulated soft thresholding can be employed to reliably reduce the portion of transform coefficients responsible for noise and preserve the sharpness of structure and object boundaries. This is important, especially for ultrasound applications, since we are most interested in identifying endocardial and epicardial boundaries. Thus we applied relatively small thresholds for wavelet shrinkage in Step 3. Our test images included some noise-induced spikes among the WCs at finer scales due to a significant amount of intensity drop caused by signal cancellation under coherent interference. We incorporated a spatial weighted averaging operation in the wavelet shrinkage operator to further diffuse the pulse energy within one or two octaves of high frequency. This effect can be seen on the screen, but may not be very obvious due to printing resolution. After wavelet shrinkage, the processed signal also looses some VE which was reflected by smoothed features. A technique intended to add signal VE to certain features was developed using a piece wise nonlinear function with hard thresholding, for removing small noise perturbations. In step 4, range-based VE gain nonlinear processing and generalized adaptive gain are utilized to enhance certain specific features of importance to cardiology. As expected, increasing signal VE at the fine scales enhanced high frequency features such as step edges while increasing signal VE at the coarse scales improved the visibility of large structures and object boundaries.

\section{EXPERIMENTAL RESULTS}

We tested our multiscale homomorphic algorithm for speckle reduction and feature enhancement on echocardiograms of varying quality. Figures 2 and 3 present the results of de-noising with or without feature enhancement on end diastolic (ED) and end systolic (ES) frames. The speckled original frames are shown first. Results from wavelet shrinkage de-noising only and de-noising with enhancement are shown in the Figures 2(b) and 2(c) respectively. Experimental results are also compared with other speckle reduction techniques, such as median filtering, extreme sharpening combined with median filtering, ${ }^{4,17}$ homomorphic Wiener filtering, and a wavelet shrinkage de-noising. ${ }^{7,6}$ Figure 4 shows sample results of the above mentioned methods on a typical frame from an echocardiographic sequence with the left ventricle as the region of interest. Figure 4(a) is sample noisy image. The result of median filtering with a 5x5 mask is shown in Figure 4(b). Figure 4(c) displays sample result of extreme sharpening combined with median filtering. The result from homomorphic Wiener filtering is shown in Figure 4(d). These last two images, 4(e) and 4(f), display the result from wavelet shrinkage de-noising only and our de-noising and enhancement algorithm. The algorithm produces smoothness inside a uniform region and contrast along structure and object boundaries in addition to speckle reduction. The de-noised and enhanced results of noisy echocardiographic images from this algorithm appear to outperform the results from soft thresholding de-noising alone. Our current algorithm is implemented such that most parameters are set dynamically for adaptive de-noising and feature enhancement. 


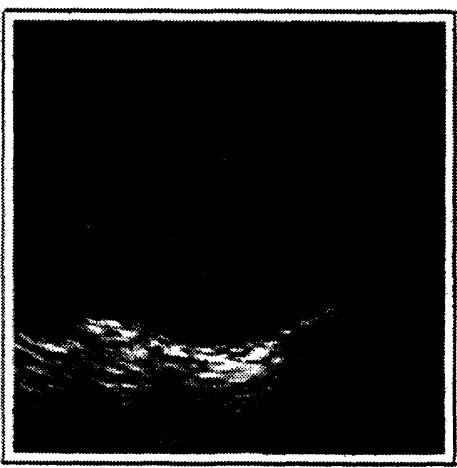

(a)

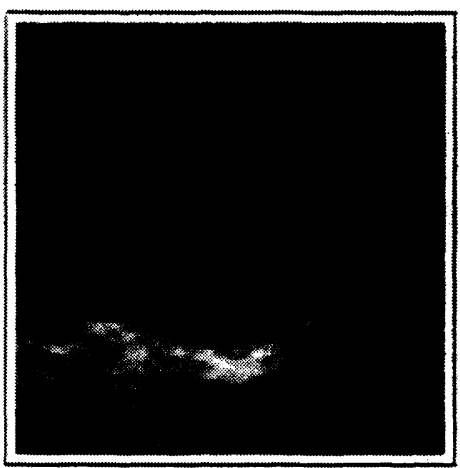

(b)

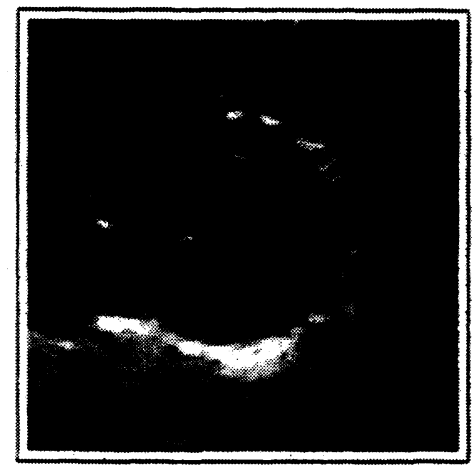

(c)

Figure 3: Results of de-noising and enhancement: (a) An noisy ES frame, (b) Wavelet shrinkage de-noising only method, and (d) DWT-based de-noising and enhancement.

\section{CLINICAL CASE STUDY}

A study of clinical images was conducted to investigate the effect of de-noising on the consistency and reliability to manually defined borders of the left ventricle in 2-D short-axis echocardiographic images by expert observers. Experimental results indicate the algorithm is promising. Borders defined by experts exhibit less variation after processing. It seems that in echocardiograms, where no real borders are clearly visible and incomplete, expert borders usually indicate a close range where real borders may occur. When two expert borders agree with each other, the range of real borders is more likely limited around the two expert borders. The study of clinical images shows that de-noising with features enhanced helps the consistency and reliability of manually defined borders by expert observers.

The set of test images included in our study of clinical images was selected from an echocardiographic database exhibiting diverse image quality. Sixty systolic sequences of 2-D short-axis echocardiographic images were selected. Half of the test images are rated as good quality while the rest were considered as poor quality. Analytic results have shown that there is some improvement in consistency and reliability for manually defined borders by expert observers examining de-noised images compared to their original noisy images. Quantitative measurements were calculated in terms of mean of absolute border differences (MDistDiff) in distance $(\mathrm{mm})$ and mean border area differences (MAreaDiff) in $\mathrm{cm}^{2}$. The border difference was measured by its close approximation in 64 radial directional difference from an estimated center ${ }^{24}$ of the left ventricle. Manually defined borders by experts looking at poor images were improved after de-noising. The statistical results of quantitative measurements of two set expert manually defined borders are shown in Table 1. The statistical computation results listed under the column "Ori" are the quantitative measurements between two sets of expert borders on the original speckled images while the results under the column "Enh" are based on the de-noised and enhanced images. It is worth mentioning that a single set of de-noising and enhancement parameters was used to process all the test echocardiographic images used in this study. We suggest that a single value set of parameters should be enough for de-noising and enhancing a class of images with a similar noise pattern and selected features.

Figure 5 shows the correlation between two sets of manually defined borders by expert observers. The four diagrams in Figure fign2(a) present the correlation of ED Epi (epicardial) border areas, ES Epi border areas, ED Endo (endocardial) border areas, and ES Endo border areas on the original noisy images. The four diagrams in Figure fign2(b) show similar results for the de-noised images with features restored or enhanced. The solid lines in the figure are the linear regression lines, while the dash and dotted lines are ideal regression lines. From the diagrams, it is clear that the points which represent the two expert border areas on the same de-noised image are, in general, more toward the ideal regression line. Additional improvement can be seen on the Endo area correlation for the de-noised images. In most echocardiograms, there is usually less Endo border information than Epi border 


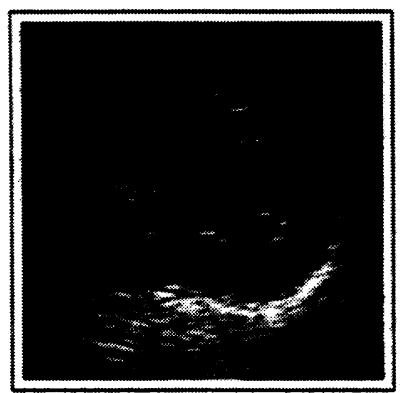

(a)

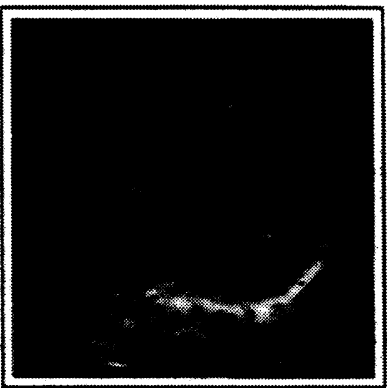

(d)

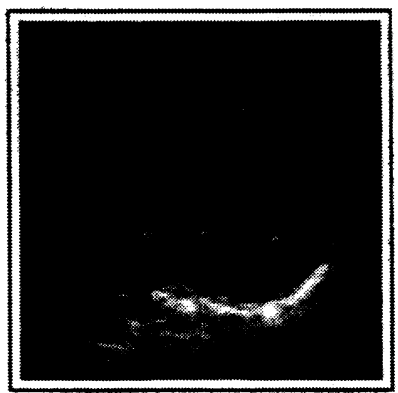

(b)

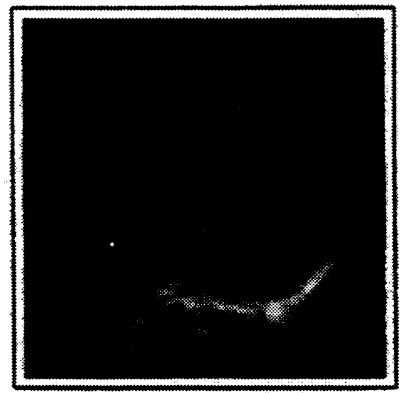

(e)

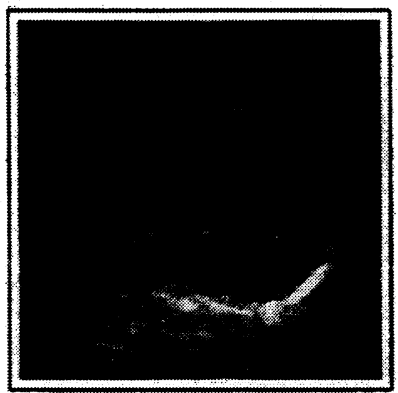

(c)

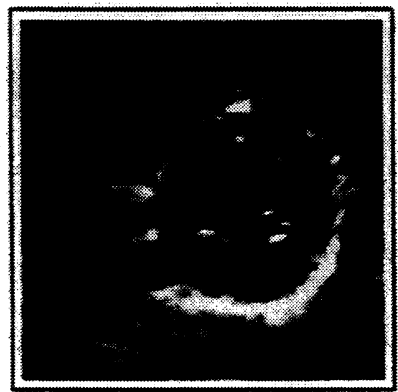

(f)

Figure 4: Results of various de-noising methods: (a) Original image with speckle noise, (b) Median filtering, (c) Extreme sharpening combined with median filtering, (d) Homomorphic Wiener filtering, (e) Wavelet shrinkage denoising only, and (f) DWT-based de-noising with enhancement.

information. Noisy border information affects border interpolation by human observers for the manually defined borders. After de-noising, Endo border information is improved, so the expert border areas tend to agree with each other, especially ES Endo areas. The statistical computation results shown in Table 1, support this analysis.

Table 1: Quantitative measurements of manually defined borders

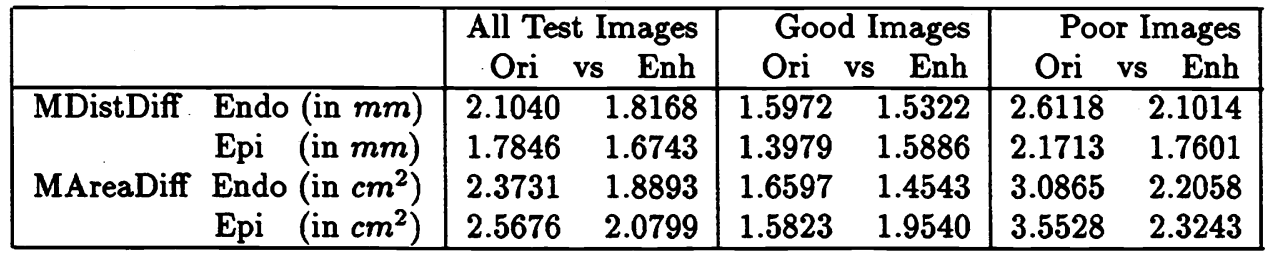

\section{CONCLUSIONS}

In this paper, we presented a multiscale homomorphic approach for speckle reduction and feature enhancement. We showed that two conflicting objectives of de-noising and enhancement can be achieved through multiscale wavelet representations. Through fine-to-coarse scale space analysis of a speckled image on a logarithmic scale, distinct behaviors of noise and features can be differentiated. We took advantage of both soft thresholding and hard thresholding wavelet shrinkage techniques. Nonlinear processing was subsequently performed for feature restoration and enhancement. The algorithm was tested by applying it to a variety of ultrasound images from an echocardiographic database exhibiting diverse image quality. A significant amount of improvement in terms of image quality was visible.

Future directions of this research may include improving de-noising and feature enhancement methods. A possible 

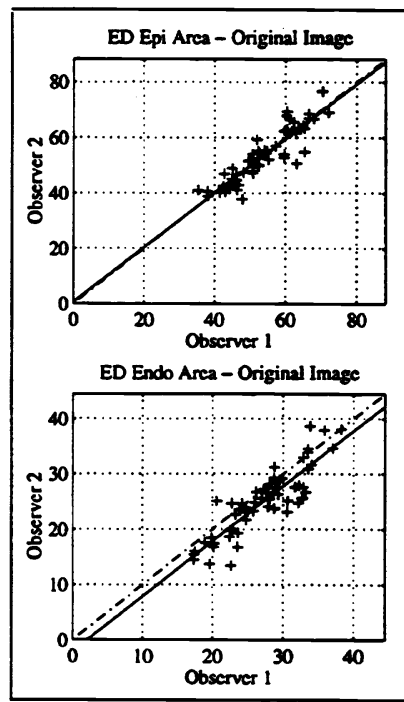
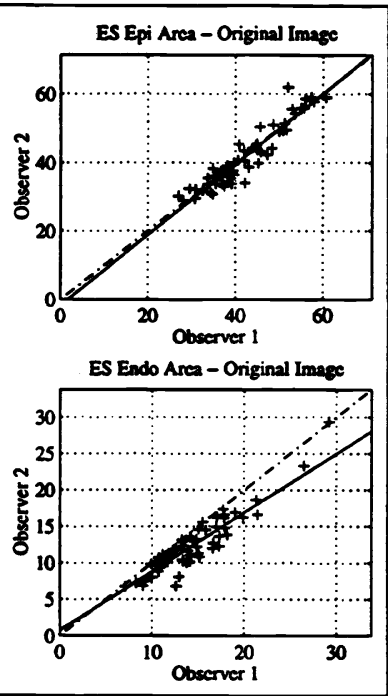

(a)
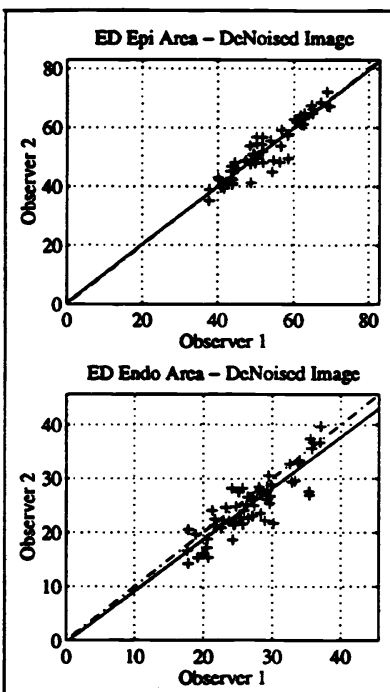
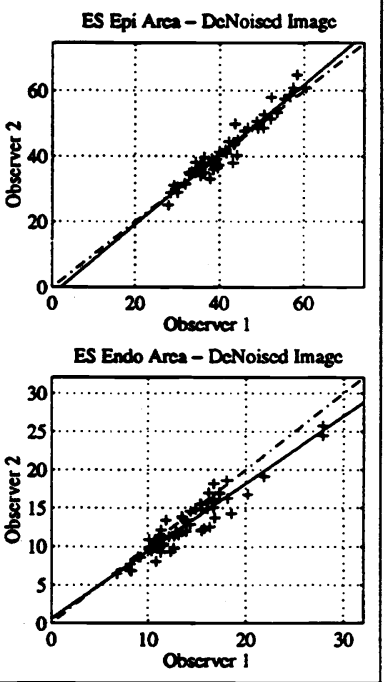

(b)

Figure 5: Correlation between manually defined borders by two expert cardiologist observers.

improvement for de-noising and enhancement might be: (1) removing isolated finer scale significant VE by utilizing the correlation of information of features within spatial-scale space, (2) finding near-optimal thresholds through coefficient and energy distributions, and (3) local maxima's correlation between scales. Other improvements may be possible through edge-based feature enhancement. ${ }^{20,14,18,19}$

\section{ACKNOWLEDGMENTS}

This research was supported in part by ATL (Advanced Technology Laboratories), Washington.

\section{REFERENCES}

1. J. G. Abbott and F. L. Thurstone, "Acoustic speckle: theory and experimental analysis," Ultrasonic Imaging, no. 1, pp. 303-324, 1979.

2. R. R. Coifman and F. Majid, "Adapted waveform analysis and denoising," Preprint, Yale University.

3. R. R. Coifman and D. L. Donoho, "Translation-invariant de-noising," Preprint, Stanford University.

4. S. M. Collins, D. J. Skorton, E. A. Geiser, J. A. Nichols, D. A. Conetta, N. G. Pandian, and R. E. Kerber, "Computer-assisted edge detection in two-dimensional echocardiography: comparison with anatomic data," Journal of the American Society of Echocardiography, vol. 53, pp. 1380-1387, 1984.

5. I. Daubechies, "Orthonormal bases of compactly supported wavelets," Commun. Pure Appl. Math., vol. XLI, pp. 909-996, 1988.

6. D. L. Donoho, "De-noising by soft-thresholding," Technical Report, Department of Statistics, Stanford University.

7. D. L. Donoho and I. M. Johnstone, "Ideal spatial adaptation via wavelet shrinkage," to appear Biometrika. 
8. E. A. Geiser, D. C. Wilson, G. L. Gibby, J. Billett, and D. A. Conetta, "A method for evaluation of enhancement operations in two-dimensional echocardiographic images," Journal of the American Society of Echocardiography, vol. 4, no. 3, pp.235-246, May, 1991.

9. E. A. Geiser, "Edge detection and wall motion analysis," Echocardiography: An International Review (ed. J. Chambers and M. J. Monaghan), pp. 71-82, Oxford University Press, Oxford, 1993.

10. J. W. Goodman, "Some fundamental properties of speckle," J. Opt. Soc. Am., vol. 66, no. 11, pp. 1145-1150, Nov., 1976.

11. H. Guo, J. E. Odegard, M. Lang, R. A. Gopinath, I. W. Selesnick, and C. S. Burrus, "Wavelet based speckle reduction with application to SAR based ATD/R," Proc. ICIP, Austin, TX, Nov., 1994.

12. J. H. Hokland and T. Taxt, "Ultrasound speckle reduction using harmonic oscillator models," IEEE Trans. on Ultrasonics, Ferroelectrics, and Frequency Control, vol. 41, no. 2, March, 1994.

13. A. K. Jain, Fundamentals of digital image processing, Prentice-Hall, Inc., Englewood Cliffs, New Jersey, 1989.

14. A. Laine, S. Song, and J. Fan, "Adaptive multiscale processing for contrast enhancement," Proceedings of SPIE: Conference on Biomedical Imaging and Biomedical Visualization, San Jose, CA, January 31-February 4, 1993.

15. A. Laine, S. Schuler, J. Fan, and W. Huda, "Mammographic feature enhancement by multiscale analysis," IEEE Trans. on Medical Imaging, vol. 13, no. 14, Dec. 1994.

16. A. Laine, J. Fan, and W. Yang, "Wavelets for contrast enhancement of digital mammography," IEEE Engineering in Medicine and Biology, vol. 14, no. 5, pp. 536-550, 1995.

17. M. J. Lester, J. F. Brenner, and W. D. Selles, "Local transforms for biomedical image analysis," Computer Graphics and Image Processing, 13, 17-30, 1980.

18. J. Lu, J. B. Weaver, D. M. Jr. Healy, and Y. Xu, "Noise reduction with multiscale edge representation and perceptual criteria," Proceedings of IEEE-SP International Symposium on Time-Frequency and Time-Scale Analysis, Victoria, BC, Oct., 1992.

19. J. Lu and D. M. Jr. Healy, "Contrast enhancement of medical images using multiscale edge representation," Proceedings of SPIE: Wavelet applications, Orlando, FL, April 5-8, 1994.

20. S. Mallat and S. Zhong, "Characterization of signals from multiscale edges," IEEE Trans. Pattern Anal. Mach. Intell., vol. 14, no. 7, pp. 710, 1992

21. S. Mallat and W. L. Hwang, "Singularity detection and processing with wavelets," IEEE Trans. Inform. Theory, vol.38, no. 2, pp. 617-643, 1992.

22. P. Moulin, "A wavelet regularization method for diffuse radar-target imaging and speckle-noise reduction," $J$. of Mathematical Imaging and Vision, no. 3, pp. 123-134, 1993.

23. L. J. Porcello, N. G. Massey, R. B. Innes, and J. M. Marks, "Speckle reduction in synthetic-aperture radars," J. Opt. Soc. Am., vol. 66, no. 11, pp. 1305-1311, Nov., 1976.

24. D. C. Wilson and E. A. Geiser, "Automatic center point determination in two-dimensional short-axis echocardiographic images," Pattern Recognition, vol. 25, no. 9, pp. 893-900, 1992. 